\section{Durable remissions in TCF3-HLF positive acute lymphoblastic leukemia with blinatumomab and stem cell transplantation}

TCF3-HLF-positive leukemia represents a rare subtype of childhood acute lymphoblastic leukemia (ALL), characterized by a high rate of treatment failure despite treatment intensification and allogeneic stem cell transplantation (SCT). Given the high and homogeneous expression of CD19 on blast cells of this leukemia subtype, these patients may benefit from CD19-directed immunotherapy. Here, we report the experience on nine TCF3-HLF-positive ALL patients, most of whom were treated early in first consolidation with blinatumomab as a bridge to SCT between 2015 and 2018. Treatment with blinatumomab was generally well tolerated; reversible neurotoxicity was observed in two patients. All nine patients achieved molecular remission after blinatumomab treatment; seven underwent SCT and for one patient SCT is planned. Median follow up after start of blinatumomab treatment was 342 days, and four patients remain in molecular remission after a follow up of 1317 , 1292, 1245, and 342 days, respectively. Three patients died because of infectious complications not directly related to blinatumomab, because they occurred either after SCT or after emergence of a CD19-negative leukemia clone. In the light of these encouraging observations, CD19-directed immunotherapy should be considered early after induction chemotherapy in TCF3-HLF-positive ALL children and patients' outcome monitored systematically by study groups.

The chromosomal translocation $\mathrm{t}(17 ; 19)$, resulting in the oncogenic fusion transcription factor TCF3-HLF, ${ }^{1}$ defines a rare cytogenetic subtype of B-cell precursor (BCP) ALL occurring in children and young adults that is associated with a dismal outcome. ${ }^{2}$ Major leukemia study groups consider TCF3-HLF-positive ALL patients eligible for addition of experimental therapies in first line. Functional screening of patient-derived xenografts revealed a dependence on BCL2 with promising response to a combination of venetoclax with vincristine and dexamethasone, ${ }^{3}$ which motivated the inclusion of a stratum allowing for combination of venetoclax with standard ALL therapy in the setting of a pediatric phase I/II study (clinicaltrials.gov identifier: 03236857). ${ }^{4}$ Moreover, given the strong homogeneous expression of CD19 in TCF3$H L F$-positive ALL, and the impressive responses to CD19-directed immunotherapeutic approaches, these patients may benefit from CD19-directed immunotherapy. ${ }^{5}$

Blinatumomab is a bispecific T-cell engager (BiTE®) antibody simultaneously binding CD3-positive cytotoxic $T$ cells and CD19-positive B cells, resulting in a T-cell mediated serial lysis of B cells. Based on promising clinical activity with effective responses in heavily pretreated patients (clinicaltrials.gov identifier: 01466179), ${ }^{6}$ blinatumomab gained accelerated approval by the US Food and Drug Administration (FDA), the European Medicines Agency (EMA), and Swissmedic agencies for treatment of both children and adults with relapsed/refractory Philadelphia chromosome-negative BCP-ALL. Treatment with blinatumomab in adults with minimal residual disease-positive $\left(\mathrm{MRD}^{+}\right)$-ALL, mostly used as a bridge to SCT, resulted in complete molecular response with MRD negativity in $80 \%$ of patients. ${ }^{7,8}$ Similarly, high rates of molecular remission were confirmed in a larger clinical study enrolling adults patients in second or later morphological complete remission (CR). ${ }^{9}$ Extensive safety and efficacy data are available from pediatric patients included into a phase I/II study and an expanded access study (RIALTO) at a recommended phase II dose of 15 $\mu \mathrm{g} / \mathrm{m}^{2} /$ day continuous infusion (clinicaltrials.gov identifier: $02187354) .{ }^{10}$ In this first study, treatment of pediatric BCP-ALL patients with relapsed/refractory disease was initiated with high disease burden, given the end point requirements of the trial, and the fact that only 16 out of 62 patients for whom MRD data were available achieved molecular CR, with a 24-month Kaplan-Meier estimate for overall survival of $25 \% .{ }^{11}$ The toxicity profile of blina-

Table 1. Clinical data of nine patients with TCF3-HLF-positive acute lymphoblastic leukemia treated with blinatumomab.

\begin{tabular}{|c|c|c|c|c|c|c|c|c|c|c|c|c|}
\hline N. & & ts' ch & acteris & & & First-line & & & & Blina & atumomab treatm & nent \\
\hline & $\begin{array}{l}\text { Age } \\
\text { (v) }\end{array}$ & Sex & $\begin{array}{l}\text { WBC } \\
\text { (nL) }\end{array}$ & $\begin{array}{l}\text { Calcium } \\
\text { (mmol/L) }\end{array}$ & EMD & $\begin{array}{l}\text { treatment } \\
\text { protocol }\end{array}$ & $\begin{array}{l}\text { Prednisone } \\
\text { response }\end{array}$ & $\begin{array}{c}\text { Disease } \\
\text { burden } \\
\text { post } \\
\text { induction }\end{array}$ & $\begin{array}{l}\text { Disease } \\
\text { status } \\
\text { prior to } \\
\text { blina }\end{array}$ & $\begin{array}{l}\text { Disease } \\
\text { involvement }\end{array}$ & SAES & $\begin{array}{l}\text { Molecular } \\
\text { remission }\end{array}$ \\
\hline 1 & 13 & $\mathrm{~F}$ & 13.8 & 2.42 & None & AALL 1131 & N/A & FACS $27.5 \%$ & $\mathrm{RD}$ & BM & Depressed LOC & Yes \\
\hline 2 & 14 & $\mathrm{~F}$ & 6.15 & 3.65 & None & AIEOP-BFM & PPR & $<10^{-3}>10^{-4}$ & Relapse & BM & $\begin{array}{c}\text { Bilateral hip } \\
\text { osteonecrosis }\end{array}$ & Yes \\
\hline 3 & 8 & M & 14.49 & 2.57 & CNS2b & AIEOP-BFM & PGR & $10^{-2}$ & MRD & BM & None & Yes \\
\hline 4 & 7 & $\mathrm{~F}$ & 5.26 & 3.2 & CNS3 & AIEOP-BFM & PGR & $6 \times 10^{-4}$ & MRD & BM & $\begin{array}{c}\text { Convulsion } \\
\text { CNS pleocytosis }^{1}\end{array}$ & Yes \\
\hline 5 & 10 & M & 4.6 & 2.46 & None & FRALLE & PGR & $5 \times 10^{-4}$ & MRD & BM & None & Yes \\
\hline 6 & 3 & $\mathrm{~F}$ & N/A & N/A & CNS2 & AIEOP-BFM & PGR & Negative & Relapse $^{2}$ & BM & None & Yes \\
\hline 7 & 8 & $\mathrm{~F}$ & 11.3 & N/A & None & AIEOP-BFM & PGR & $7.5 \times 10^{-3}$ & MRD & BM & None & Yes \\
\hline 8 & 5 & $\mathrm{~F}$ & 25.7 & 2.63 & None & UKALL & $\mathrm{N} / \mathrm{A}$ & $3 \times 10^{-5}$ & MRD & $\mathrm{BM}$ & None & Yes \\
\hline 9 & 7 & M & 8.2 & 5.03 & None & AIEOP-BFM & PGR & $3.2 \times 10^{-3}$ & MRD & BM & CRS & $\mathrm{Yes}^{4}$ \\
\hline
\end{tabular}

Blina: blinatumomab;WBC: white blood cell count; EMD: extramedullary disease; RD: refractory disease; BM: bone marrow; SAE: serious adverse events; y: years; LOC: loss of consciousness; CRS: cytokine release syndrome. ${ }^{1}$ Central nervous system (CNS) pleocytosis without disease involvement. ${ }^{2}$ Initial diagnosis was c-acute lymphoblastic leukemia (ALL), isolated relapse under maintenance TCF3-HLF positive. ${ }^{3}$ Molecular remission defined as per Online Supplementary Table S1. ${ }^{4}$ One marker positive nonquantifiable; one marker negative after one cycle blinatumomab. 
Table 2. Definitive treatment of nine patients with blinatumomab and outcome.

\begin{tabular}{|c|c|c|c|c|c|c|}
\hline N. & $\begin{array}{l}\text { Donor type } \\
\text { for HSCT }\end{array}$ & Conditioning & $\begin{array}{c}\text { HSCT } \\
\text { GvHD } \\
\text { prophylaxis }\end{array}$ & GvHD & $\begin{array}{l}\text { Current } \\
\text { status }\end{array}$ & $\begin{array}{l}\text { FU } \\
\text { time }(\text { di) }\end{array}$ \\
\hline 1 & $\begin{array}{l}\text { Matched } \\
\text { Unrelated }\end{array}$ & Bu, Thiotepa, Cy & $\begin{array}{c}\text { CsA } \\
\text { GvHD }\end{array}$ & Mild & ACR & 1317 \\
\hline 2 & $\begin{array}{c}\text { Matched } \\
\text { Unrelated }^{1}\end{array}$ & $\begin{array}{l}\text { Bu, Cy, VP16, } \\
\text { Thymoglobulin }\end{array}$ & $\mathrm{CsA}$ & $\begin{array}{l}\text { Mild } \\
\text { GvHD }\end{array}$ & ACR & 1292 \\
\hline 3 & $\begin{array}{c}\text { Matched } \\
\text { Unrelated }\end{array}$ & TBI, VP16, ATG & CsA, MTX & $\begin{array}{c}\text { Moderate } \\
\text { GvHD }\end{array}$ & ACR & 1245 \\
\hline 4 & Haploidentical $^{2}$ & $\begin{array}{l}\text { Melphalan, } \\
\text { Thiotepa, } \\
\text { Fludarabine }\end{array}$ & $\begin{array}{l}\text { Alemtuzumab, } \\
\text { MM }\end{array}$ & No & $\begin{array}{l}\text { Died from infection } \\
\text { under remission }\end{array}$ & 363 \\
\hline 5 & No HSCT & - & - & - & $\begin{array}{c}\text { Died from infection } \\
\text { under CD19-neg relapse }\end{array}$ & 210 \\
\hline 6 & $\begin{array}{l}\text { Matched } \\
\text { Related }\end{array}$ & TBI, VP16 & CsA, MTX & $\begin{array}{c}\text { Moderate } \\
\text { GvHD }\end{array}$ & $\begin{array}{l}\text { Relapse; died from } \\
\text { infection after HSCT }\end{array}$ & 120 \\
\hline 7 & Haploidentical & $\begin{array}{l}\text { TBI, Thiotepa, } \\
\text { Fludarabine }\end{array}$ & None & No & ACR & 342 \\
\hline 8 & $\begin{array}{c}\text { Matched } \\
\text { Unrelated }\end{array}$ & TBI, Cy & Alemtuzumab, CsA, MTX & No & ACR & 160 \\
\hline 9 & No HSCT & - & - & - & ACR & 79 \\
\hline
\end{tabular}

FU: follow up; neg: negative; MM: mycophenolate mofetil;ACR: alive and in complete remission. ${ }^{~}$ Patient underwent prior transplantation after induction but relapsed shortly after. ${ }^{2}$ Patient underwent prior transplantation after 2 cycles blinatumomab, but relapsed shortly after. ${ }^{3}$ Follow up since first day of blinatumomab treatment. HSCT: hematopoietic stem cell transplantation; Bu: buprenorphin; ATG: Antithymocyte globulin; Bu: busulfan; Cy: cyclophosphamide; VP16: etoposide; TBI: total body irradiation CsA: Cyclosporine A; MTX: methotrexate; GvHD: Graft-versus-host disease.

tumomab is well established, including cytokine release syndrome (CRS) and reversible neurological events, such as ataxia, seizures, and encephalopathy. The mechanisms leading to neurotoxicity remain unclear and may relate to variable expression of CD19 within the brain. Given the good tolerability and promising efficacy data, the potential benefit of blinatumomab in improving outcome is being investigated in patients experiencing high-risk ALL first relapse (clinicaltrials.gov identifier: 02393859) and will be evaluated in first-line treatment in patients with intermediate and high-risk BCP-ALL by the Italian Association of Pediatric Hematology and Oncology-Berlin-FrankfurtMuenster (AIEOP-BFM) study group (clinicaltrials.gov identifier: 02393859).

Here, we report the European experience with blinatumomab using retrospectively collected data from nine patients with TCF3-HLF-positive ALL treated with blinatumomab between 2015 and 2018 by members of the international BFM study group after approval by the competent ethics committee; two of these nine patients were enrolled in the expanded access RIALTO protocol into Relapsed/Refractory B-precursor ALL. An overview of patients' data and clinical courses is provided in Tables 1 and 2, and Figure 1.

Blinatumomab was initiated for disease refractory to induction chemotherapy (patient 1), for persistent MRD after consolidation chemotherapy (patients 3, 4, 5, 7, 8, 9 ), and after first relapse (patients 2,6). Blinatumomab was administered at $15 \mu \mathrm{g} / \mathrm{m}^{2} /$ day for $2-4$ cycles. All nine patients responded, obtaining molecular CR after either the first cycle (8 out of 9) or the second cycle (patient 7). Seven out of nine patients underwent allogeneic SCT after blinatumomab treatment. Details on the type of donor are shown in Table 2. Four patients developed mild to moderate acute graft-versus-host disease (GvHD). No suitable donor could be found in time for patient 5, and this patient relapsed with CD19-negative leukemia blasts.

Four patients achieved a long-term remission and are still MRD negative after a follow up of 1317, 1292, 1245, and 342 days, respectively (Figure 1). Given the expected risk for subsequent relapse, additional therapy was provided after SCT with one (patient 4) or two (patients 2 and 3) cycles of blinatumomab. Three patients died from infectious complications: patient 4 due to a transplantrelated adenoviral infection while in second molecular remission after her second SCT, patient 5 from a fungal infection while in CD19-negative relapse, and patient 6 from transplant-related aspergillus infection while in molecular relapse. Significant neurotoxicity occurred in two patients (patients 1 and 4). For patient 1, blinatumomab had to be interrupted due to a state of mental confusion and a decreased level of consciousness lasting 24 hours; it was resumed with a reduced dose of 5 $\mu \mathrm{g} / \mathrm{m}^{2} /$ day, and was finally stopped because of seizures. This patient is currently still in molecular remission after SCT. Similar complications have previously been reported in pediatric patients. ${ }^{12,13}$ Disseminated intravascular coagulation was reported during treatment in two patients with overt leukemia, but was not considered to be treatment-related because TCF3-HLF-positive leukemia often presents with coagulopathies. Patient 9 developed grade 2 CRS lasting for 48 hours. No other significant toxicity was reported. 
This is the first report of clinical activity of CD19directed immunotherapy in TCF3-HLF-positive ALL, a rare BCP-ALL subtype reported to be almost always fatal even with the most intensive conventional treatment regimens. ${ }^{2}$ Although the number of patients treated is small and follow-up time limited, these results provide a strong rationale for rapid intervention with immunotherapy for this ALL subtype. The response to blinatumomab has been reported to be less effective in patients with higher disease burden, suggesting that this approach may be more effective in the MRD setting. ${ }^{6}$ Current ongoing randomized pediatric studies will hopefully provide more evidence to answer this question. Until then, we recommend using a different treatment modality to reduce the leukemia burden before initiating blinatumomab. A conventional cytoreduction will also be required if immunotherapy with autologous chimeric antigen receptor $\mathrm{T}(\mathrm{CAR}-\mathrm{T})$ cell is considered, allowing lymphapheresis and CAR-T-cell manufacturing. Based on the current evidence, blinatumomab appears to be a promising therapeutic element to improve the quality of remission in TCF3-HLF-positive BCP-ALL patients as a bridge to SCT. In contrast, CAR-T might provide an attractive stand-alone treatment for definitive therapy without SCT. In general, this approach will have to be validated in clinical studies. Given the strong preclinical evidence for sensitivity to the BCL2 inhibitor venetoclax in most cases, including relapsing patients, combination of venetoclax with standard ALL chemotherapy may provide an additional treatment element to improve outcome in these patients. Patients could be evaluated by functional screening using drug response profiling ${ }^{3,14}$ and $\mathrm{BH} 3$ profiling $^{15}$ at diagnosis. Three patients in this cohort have received additional courses of blinatumomab post transplant for further consolidation of the leukemia remission, since even with MRD-negative remissions prior to and after allogeneic HSCT relevant rates of subsequent relapses are observed in TCF3-HLF-positive ALL. ${ }^{2}$
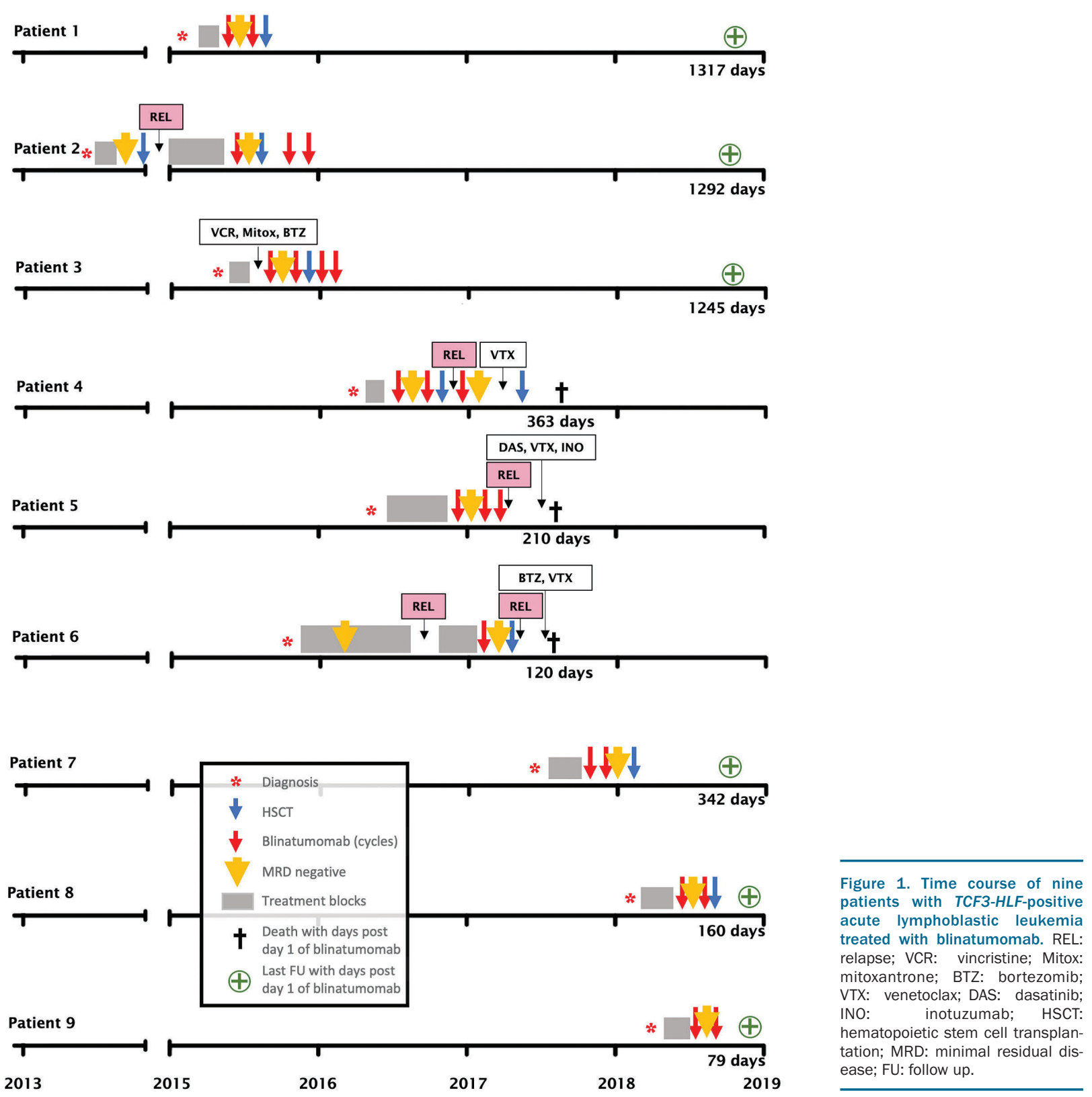
Persisting clones in compartments other than bone marrow may not be covered by MRD quantification but could still be responsive to blinatumomab therapy. The responses detected in this TCF3-HLF-positive ALL cohort are encouraging and suggest that the application of immunotherapy prior to extensive clonal selection secondary to intensive chemotherapy may be beneficial. As data are updated, the true value of this approach can be assessed. The benefit of adding blinatumomab to frontline ALL chemotherapy will be addressed in an international prospective clinical trial (clinicaltrials.gov identifier: 03643276). Taken together, our results indicate that immunotherapy may improve the outcome of TCF3-HLF-positive ALL.

Brice Mouttet, ${ }^{1}$ Luciana Vinti, ${ }^{2}$ Philip Ancliff, ${ }^{3}$

Nicole Bodmer, ${ }^{1}$ Benoit Brethon, ${ }^{4}$ Gunnar Cario, ${ }^{5}$ Christiane Chen-Santel, ${ }^{6}$ Sarah Elitzur, ${ }^{7}$ Volkan Hazar, ${ }^{8}$ Joachim Kunz,, Anja Möricke, Jerry Stein, ${ }^{10}$ Ajay Vora, ${ }^{3}$ Yöntem Yaman, ${ }^{8}$ Martin Schrappe, ${ }^{5,11}$ Sema Anak, ${ }^{8}$ André Baruchel, ${ }^{4}$ Franco Locatelli, Arend von Stackelberg, ${ }^{6}$ Martin Stanulla ${ }^{12}$ and Jean-Pierre Bourquin

'Pediatric Oncology, University Children's Hospital Zurich, Switzerland; ${ }^{2}$ Department of Pediatric Haemato-Oncology, IRCCS Ospedale Bambino Gesù, Sapienza University of Rome, Italy; ${ }^{3}$ Haematology and Oncology Department, Great Ormond Street Hospital, London, UK; ${ }^{4}$ Department of Pediatric Hematology, Hôpital Robert Debré, Assistance Publique - Hôpitaux de Paris, France; 'Department of Pediatrics I, ALL-BFM Study Group, ChristianAlbrechts University Kiel and University Medical Center SchleswigHolstein, Kiel, Germany; ${ }^{6}$ Department of Pediatric Oncology/Hematology, Charité Universitätsmedizin, Berlin, Germany; 'Pediatric Hematology-Oncology, Schneider Children's Medical Center, Sackler Faculty of Medicine, Tel Aviv University, Israel; 'Department of Pediatric Hematology, Medipol University Hospital, Istanbul, Turkey; ${ }^{9}$ Pediatric Oncology, Hematology and Immunology, University of Heidelberg, Germany; ${ }^{10}$ Bone Marrow Transplant Unit, Schneider Children's Medical Center, Sackler Faculty of Medicine, Tel Aviv University, Israel; "Pediatrics, Christian Albrechts University Kiel, University Medical Center, Kiel, Germany and '2Pediatric Hematology and Oncology, Hannover Medical School, Germany

Correspondence: JEAN-PIERRE BOURQUIN. jean-pierre.bourquin@kispi.uzh.ch doi:10.3324/haematol.2018.210104

Acknowledgments: the authors thank Yun Huang (University Children's Hospital Zurich) for careful review of the manuscript and Renate Siegenthaler (University Children's Hospital Zurich) to provide data.

Information on authorship, contributions, and financial \& other disclosures was provided by the authors and is available with the online version of this article at wWw. haematologica.org.

\section{References}

1. Inaba T, Roberts WM, Shapiro LH, et al. Fusion of the leucine zipper gene HLF to the E2A gene in human acute B-lineage leukemia. Science. 1992;257(5069):531-534.

2. Minson KA, Prasad P, Vear S, et al. t(17;19) in Children with Acute Lymphocytic Leukemia: A Report of 3 Cases and a Review of the Literature. Case Rep Hematol. 2013;2013(4):563291-563294.

3. Fischer U, Forster M, Rinaldi A, et al. Genomics and drug profiling of fatal TCF3-HLF-positive acute lymphoblastic leukemia identifies recurrent mutation patterns and therapeutic options. Nat Genet. 2015;47(9):1020-1029.

4. Place AE, Goldsmith K, Bourquin J-P, et al. Accelerating drug development in pediatric cancer: a novel Phase I study design of venetoclax in relapsed/refractory malignancies. Future Oncol. 2018;14(21):2115-2129.

5. Velasquez MP, Bonifant CL, Gottschalk S. Redirecting T cells to hematological malignancies with bispecific antibodies. Blood. 2018;131(1):30-38.

6. Topp MS, Gökbuget N, Stein AS, et al. Safety and activity of blinatumomab for adult patients with relapsed or refractory B-precursor acute lymphoblastic leukaemia: a multicentre, single-arm, phase 2 study. Lancet Oncol. 2015;16(1):57-66.

7. Topp MS, Kufer P, Gökbuget N, et al. Targeted Therapy With the TCell-Engaging Antibody Blinatumomab of ChemotherapyRefractory Minimal Residual Disease in B-Lineage Acute Lymphoblastic Leukemia Patients Results in High Response Rate and Prolonged Leukemia-Free Survival. J Clin Oncol. 2011;29(18):24932498.

8. Topp MS, Gökbuget N, Zugmaier G, et al. Long-term follow-up of hematologic relapse-free survival in a phase 2 study of blinatumomab in patients with MRD in B-lineage ALL. Blood. 2012;120(26):5185-5187.

9. Gökbuget N, Dombret H, Bonifacio M, et al. Blinatumomab for minimal residual disease in adults with B-cell precursor acute lymphoblastic leukemia. Blood. 2018;131(14):1522-1531.

10. Stackelberg von A, Locatelli F, Zugmaier G, et al. Phase $1 / 2$ Study in Pediatric Patients with Relapsed/Refractory B-Cell Precursor Acute Lymphoblastic Leukemia (BCP-ALL) Receiving Blinatumomab Treatment. Blood. 2014;124(21):2292.

11. Gore L, Locatelli F, Zugmaier G, et al. Survival after blinatumomab treatment in pediatric patients with relapsed/refractory B-cell precursor acute lymphoblastic leukemia. Blood Cancer J. 2018;8(9):80.

12. Teachey DT, Rheingold SR, Maude SL, et al. Cytokine release syndrome after blinatumomab treatment related to abnormal macrophage activation and ameliorated with cytokine-directed therapy. Blood. 2013;121(26):5154-5157.

13. Stackelberg von A, Locatelli F, Zugmaier G, et al. Phase I/Phase II Study of Blinatumomab in Pediatric Patients With Relapsed/Refractory Acute Lymphoblastic Leukemia. J Clin Oncol 2016;34(36):4381-4389.

14. Frismantas V, Dobay MP, Rinaldi A, et al. Ex vivo drug response profiling detects recurrent sensitivity patterns in drug-resistant acute lymphoblastic leukemia. Blood. 2017;129(11):e26-e37.

15. Deng J, Carlson N, Takeyama K, Dal Cin P, Shipp M, Letai A. BH3 Profiling Identifies Three Distinct Classes of Apoptotic Blocks to Predict Response to ABT-737 and Conventional Chemotherapeutic Agents. Cancer Cell. 2007;12(2):171-185. 